\title{
Limiting Behavior of the Partial Sum for Negatively Superadditive Dependent Random Vectors in Hilbert Space
}

\author{
Mi-Hwa Ko \\ Division of Mathematics and Informational Statistics, Wonkwang University, Jeonbuk 54538, Republic of Korea \\ Correspondence should be addressed to Mi-Hwa Ko; songhack@wonkwang.ac.kr
}

Received 25 May 2020; Accepted 3 August 2020; Published 24 August 2020

Academic Editor: Ghulam Shabbir

Copyright (C) 2020 Mi-Hwa Ko. This is an open access article distributed under the Creative Commons Attribution License, which permits unrestricted use, distribution, and reproduction in any medium, provided the original work is properly cited.

In this paper, We study the complete convergence and $L_{p^{-}}$convergence for the maximum of the partial sum of negatively superadditive dependent random vectors in Hilbert space. The results extend the corresponding ones of Ko (Ko, 2020) to $H$-valued negatively superadditive dependent random vectors.

\section{Introduction}

Alam and Saxena [1] introduced the concept of negative association as follows: a finite family of random variables $\left\{X_{i}, 1 \leq i \leq n\right\}$ is said to be negatively associated (NA) for every pair of disjoint subsets of $A$ and $B$ of $\{1,2, \ldots, n\} \operatorname{Cov}\left(f\left(X_{i}, i \in A\right) g\left(X_{j}, j \in B\right)\right) \leq 0$, whenever $f$ and $g$ are coordinate-wise nondecreasing functions and the covariance exists. An infinite family is NA if every finite subfamily is NA.

A function $\phi: \mathbb{R}^{n} \longrightarrow \mathbb{R}$ is said to be superadditive if $\phi(x \vee y)+\phi(x \wedge y) \geq \phi(x)+\phi(y)$ for all $x, y \in \mathbb{R}^{n}$, where $\vee$ is for componentwise maximum and $\Lambda$ is for componentwise minimum. This notion was introduced by Kemperman [2].

Based on the above superadditive function, the concept of negatively superadditive dependent (NSD) random variables was introduced by $\mathrm{Hu}$ [3] as follows: a random vector $X=\left(X_{1}, X_{2}, \ldots, X_{n}\right)$ is said to be negatively superadditive dependent (NSD) if $E \phi\left(X_{1}, X_{2}, \ldots, X_{n}\right) \leq E \phi\left(X_{1}^{*}\right.$, $\left.X_{2}^{*}, \ldots, X_{n}^{*}\right)$, where $X_{1}^{*}, X_{2}^{*}, \ldots, X_{n}^{*}$ are independent such that $X_{i}^{*}$ and $X_{i}$ have the same distribution for each $i$ and $\phi$ is a superadditive function such that the above expectations exist. A sequence $\left\{X_{n}, n \geq 1\right\}$ of random variables is said to be NSD if for any $n \geq 1,\left\{X_{1}, X_{2}, \ldots, X_{n}\right\}$ is NSD. Hu [3] gave an example for illustrating that negatively superadditive dependence (NSD) does not imply negative association (NA), and Christofides and Vaggelatou [4] indicated that NA implies NSD. So, the NSD structure is an extension of the NA structure. Sometimes, the NSD structure is more useful than the NA structure and NSD random variables have wide applications in reliability theory and multivariate statistical analysis. For this reason, studying the limit theorems for NSD random variables is much significant.

Let $H$ be a real separable Hilbert space with the norm $\|\cdot\|$ generated by an inner product $\langle\cdot, \cdot\rangle$. Let $\left\{e_{j}, j \geq 1\right\}$ be an orthonormal basis in $H, X$ be an $H$-valued random vector, and $\left\langle X, e_{j}\right\rangle$ be denoted by $X^{(j)}$.

Ko et al. [5] and Huan et al. [6] introduced the concept of negatively associated random vectors taking values in $H$, Ko [7] introduced the concept of asymptotically negatively associated $H$-valued random vectors, and Dung et al. [8] introduced the concept of pairwise NQD $H$-valued random vectors.

As the concept of $H$-valued NA random vectors was introduced by Ko et al. [5], Son et al. [9] presented the concept of $H$-valued negatively superadditive dependent (NSD) random vectors as follows: a sequence $\left\{X_{n}, n \geq 1\right\}$ of $H$-valued random vectors is said to be NSD if for any $d \geq 1$, the sequence $\left\{\left(X_{n}^{(1)}, X_{n}^{(2)}, \ldots, X_{n}^{(d)}\right), n \geq 1\right\}$ of $\mathbb{R}^{d}$-valued random vectors is negatively superadditive dependent (NSD).

Let $\left\{X, X_{n}, n \geq 1\right\}$ be a sequence of $H$-valued random vectors. We will use the following inequalities: 


$$
C_{1} P(\|X\|>t) \leq \frac{1}{n} \sum_{k=1}^{n} P\left(\left\|X_{k}\right\|>t\right) \leq C_{2} P(\|X\|>t) .
$$

If there exists a positive constant $C_{1}\left(C_{2}\right)$ such that the left-hand side (right-hand side) of (1) is satisfied for all $n \geq 1$ and $t \geq 0$, then the sequence $\left\{X_{n}, n \geq 1\right\}$ is said to be weakly lower (upper) bounded by $X$. The sequence $\left\{X_{n}, n \geq 1\right\}$ is said to be weakly bounded by $X$ if it is both lower and upper bounded by $X$.

In this paper, we show the complete convergence results and $L_{2}$-convergence of the maximum of the partial sums for NSD random vectors in Hilbert space. We also consider residual Cesàro alpha-integrability and strongly residual Cesàro alpha-integrability for NSD random vectors in Hilbert space.

Throughout the paper, the symbol $C$ denotes a generic constant which is not necessarily the same in each occurrence, and denotes the $L_{2}$-norm. Moreover, « represents the Vinogradov symbol $O$ and $I(\cdot)$ is the indicator function.

\section{Some Lemmas}

The following lemmas will be useful to prove the main results.

Lemma 1 (see [3]). If $\left(X_{1}, X_{2}, \ldots, X_{n}\right)$ is negatively superadditive dependent (NSD) and $f_{1}, f_{2}, \ldots, f_{n}$ are all nondecreasing functions, then $\left(f_{1}\left(X_{1}\right), f_{2}\left(X_{2}\right)\right.$, $\left.\ldots, f_{n}\left(X_{n}\right)\right)$ is also NSD.

Lemma 2 (see [10]). Let $\left\{X_{n}, n \geq 1\right\}$ be a sequence of NSD random variables with $E X_{n}=0$ and $E X_{n}^{2}<\infty$ for all $n \geq 1$. Then, for all $n \geq 1$, there is a positive constant $C$ such that

$$
E \max _{1 \leq k \leq n}\left(\sum_{i=1}^{k} X_{i}\right)^{2} \leq C \sum_{i=1}^{n} E X_{i}^{2} \text {. }
$$

We extend Lemma 2 to a sequence of Hilbert valued random vectors as follows.

Lemma 3. Let $\left\{X_{n}, n \geq 1\right\}$ be a sequence of $H$-valued NSD random vectors with $E X_{n}=0$ and $E\left\|X_{n}\right\|^{2}<\infty$ for every $n \geq 1$, where $X_{n}=\left(X_{n}^{(1)}, X_{n}^{(2)}, \ldots, X_{n}^{(j)}\right)$ for any $j \geq 1$ and $X_{n}^{(j)}=\left\langle X_{n}, e_{j}\right\rangle$. If $\left\{X_{n}^{(j)}, n \geq 1\right\}$ is a sequence of NSD random variables with $E X_{n}^{(j)}=0$ and $E\left(X_{n}^{(j)}\right)^{2}<\infty$ for each $j \geq 1$, then there is a positive constant $C$ such that

$$
E \max _{1 \leq k \leq n}\left\|\sum_{i=1}^{k} X_{i}\right\|^{2} \leq C \sum_{i=1}^{n} E\left\|X_{i}\right\|^{2} .
$$

Proof. Inspired by the proof of Lemma 1.7 of Huan et al. [6] and from Lemma 2, we have that

$$
\begin{aligned}
E\left(\max _{1 \leq k \leq n}\left\|\sum_{i=1}^{k} X_{i}\right\|^{2}\right) & =E\left(\max _{1 \leq k \leq n} \sum_{j=1}^{\infty}\left(<\sum_{i=1}^{k} X_{i}, e_{j}>\right)^{2}\right) \\
& \leq E\left(\sum_{j=1}^{\infty} \max _{1 \leq k \leq n}\left(<\sum_{i=1}^{k} X_{i}, e_{j}>\right)^{2}\right) \\
& =\sum_{j=1}^{\infty} E \max _{1 \leq k \leq n}\left(\sum_{i=1}^{k} X_{i}^{(j)}\right)^{2} \\
& \leq C \sum_{j=1}^{\infty} \sum_{i=1}^{n} E\left|X_{i}^{(j)}\right|^{2}=C \sum_{i=1}^{n} E\left\|X_{i}\right\|^{2} .
\end{aligned}
$$

It is obvious that if $\left\{X_{n}, n \geq 1\right\}$ is an NSD sequence of $H$-valued random vectors, where $X_{n}=\left(X_{n}^{(1)}, X_{n}^{(2)}, \ldots, X_{n}^{(j)}\right)$ for any $j \geq 1$, then $\left\{X_{n}^{(j)}\right\}$ is a sequence of NSD random variables for each $j \geq 1$. However, the reverse is not true in general.

Lemma 4 (see [11]). Let $\left\{a_{n}, n \geq 1\right\}$ and $\left\{b_{n}, n \geq 1\right\}$ be sequences of non-negative numbers. If $\sup ^{-1} \sum_{i=1}^{n} a_{i}<$ $\infty$ and $\sum_{n=1}^{\infty} b_{n}<\infty, \quad$ then $\quad \sum_{i=1}^{n} a_{i} b_{i} \leq\left(\operatorname{setp}_{m \geq 1} m^{-1} \sum_{i=1}^{m} a_{i}\right)$ $\sum_{i=1}^{n} b_{i}$ for every $n \geq 1$.

Lemma 5 (see [12]). Let $\left\{X_{n}, n \geq 1\right\}$ be a sequence of $H$-valued random vectors, weakly upper bounded by a random vector $X$. Let $r>0$ for some $A>0$.

$$
\begin{aligned}
& X_{i}^{\prime}=X_{i} I\left(\left\|X_{i}\right\| \leq A\right), \\
& X_{i}^{\prime \prime}=X_{i} I\left(\left\|X_{i}\right\|>A\right), \\
& X^{\prime}=X I\left(\left\|X_{i}\right\| \leq A\right), \\
& X^{\prime \prime}=X I(\|X\|>A) .
\end{aligned}
$$

And
(i) If $E\|X\|^{r}<\infty$, then $(1 / n) \sum_{i=1}^{n} E\left\|X_{i}\right\|^{r} \leq C E\|X\|^{r}$
(ii) $(1 / n) \sum_{i=1}^{n} E\left\|X_{i}^{\prime}\right\|^{r} \leq C\left(E\|X \prime\|^{r}\right)+A^{r} P(\|X\|>A)$
(iii) $(1 / n) \sum_{i=1}^{n} E\left\|X_{i}^{\prime \prime}\right\|^{r} \leq C E\left\|X^{\prime \prime}\right\|^{r}$

\section{Main Results}

A sequence $\left\{X_{n}, n \geq 1\right\}$ of random vectors is said to converge completely to a constant $C$ if for any $\varepsilon>0$, $\sum_{n=1}^{\infty} P\left(\left\|X_{n}-C\right\|>\varepsilon\right)<\infty$.

In this case, we write $X_{n} \longrightarrow C$ completely. This notion was given by $\mathrm{Hsu}$ and Robbins [13]. Note that complete convergence implies the almost sure convergence in view of the Borel-Cantelli lemma.

Based on Lemma 3, we will extend the complete convergence results of the maximum of the partial sum of NSD random variables to the case of $H$-valued random vectors. 
Theorem 1. Let $\left\{X_{n}, n \geq 1\right\}$ be a sequence of $H$-valued NSD random vectors. If a sequence $\left\{X_{n}, n \geq 1\right\}$ satisfies

$$
\sup _{n \geq 1} \frac{1}{n} \sum_{k=1}^{n} E\left\|X_{k}\right\|^{2}<\infty,
$$

then for any $\delta>(1 / 2)$,

$$
n^{-\delta} \max _{1 \leq i \leq n}\left\|S_{i}-E S_{i}\right\| \longrightarrow 0 \text { completely, }
$$

where $S_{i}=\sum_{j=1}^{i} X_{j}$.

Proof. For each $n \geq 1$, let $m=m_{n}$ be the integer such that $2^{m-1}<n \leq 2^{m}$. Then, we obtain that

$$
\begin{aligned}
n^{-\delta} \max _{1 \leq i \leq n}\left\|S_{i}-E S_{i}\right\| & \leq n^{-\delta} \max _{1 \leq i \leq 2^{m}}\left\|S_{i}-E S_{i}\right\| \\
& \leq\left(2^{m-1}\right)^{--\delta} \max _{1 \leq i \leq 2^{m}}\left\|S_{i}-E S_{i}\right\| \\
& =2^{\delta} \cdot 2^{-m \delta} \max _{1 \leq i \leq 2^{m}}\left\|S_{i}-E S_{i}\right\| .
\end{aligned}
$$

Hence, it is enough to prove

$$
2^{-m \delta} \max _{1 \leq i \leq 2^{m}}\left\|S_{i}-E S_{i}\right\| \longrightarrow 0 \text { completely. }
$$

By Lemma 3, the Hölder inequality, Lemma 4, and (6), we have that

$$
\begin{aligned}
\sum_{m=0}^{\infty} E\left(2^{-m \delta} \max _{1 \leq i \leq 2^{m}}\left\|S_{i}-E S_{i}\right\|\right)^{2} & \ll \sum_{m=0}^{m=0} 2^{-2 m \delta} \sum_{i=1}^{2^{m}} E\left\|X_{i}\right\|^{2} \\
& \ll \sum_{i=1}^{\infty} E\left\|X_{i}\right\|^{2} \sum_{\left\{m: 2^{m} \geq i\right\}} 2^{-2 m \delta} \leq \sum_{i=1}^{\infty} i^{-2 \delta} E\left\|X_{i}\right\|^{2} \\
& \leq\left(\sup _{n \geq 1} \frac{1}{n} \sum_{i=1}^{n} E\left\|X_{i}\right\|^{2}\right) \sum_{n=1}^{\infty} n^{-2 \delta} \ll \sum_{n=1}^{\infty} n^{-2 \delta}<\infty
\end{aligned}
$$

which yields (9). Hence, the desired result (7) follows.

Based on Lemma 3, we will extend some $L_{2}$-convergence of NSD random variables to the case of $H$-valued random vectors.

Theorem 2. Let $\left\{X_{n}, n \geq 1\right\}$ be a sequence of $H$-valued NSD random vectors satisfying (6); then, for any $\delta>(1 / 2)$,

$$
n^{-\delta} \max _{1 \leq i \leq n}\left\|S_{i}-E S_{i}\right\| \longrightarrow 0 \text { in } L_{2} \text {. }
$$

Proof. Let $X_{n}=\left(X_{n}^{(1)}, X_{n}^{(2)}, \ldots, X_{n}^{(j)}\right)$ for any $j \geq 1$. Note that $\left\{X_{n}^{(j)}\right\}$ for any $j \geq 1$ is a sequence of NSD random variables. Then, $\left\{X_{n}^{(j)}-E X_{n}^{(j)} n \geq 1\right\}$ is a sequence of $H$-valued NSD random variables by Lemma 1 . By Lemma 3, Hölder's inequality, and (6), we obtain

$$
\begin{aligned}
E\left(n^{-\delta} \max _{1 \leq i \leq n}\left\|S_{i}-E S_{i}\right\|\right)^{2} & =n^{-2 \delta} E \max _{1 \leq i \leq n}\left\|\sum_{j=1}^{i}\left(X_{j}-E X_{j}\right)\right\|^{2} \\
& \ll n^{-2 \delta} \sum_{i=1}^{n} E\left\|X_{i}-E X_{i}\right\|^{2} \\
& \ll n^{-2 \delta} \sum_{i=1}^{n} E\left\|X_{i}\right\|^{2} \ll n^{-2 \delta+1} \\
& \cdot\left(\sup _{n \geq 1} \frac{1}{n} \sum_{i=1}^{n} E\left\|X_{i}\right\|^{2}\right),
\end{aligned}
$$

which yields (11) for any $\delta>(1 / 2)$. The proof of theorem is completed.

We consider $L_{2}$-convergence of weakly upper bounded $H$-valued NSD random vectors.

Theorem 3. Let $\left\{X_{n}, n \geq 1\right\}$ be a sequence of $H$-valued NSD random vectors which is weakly upper bounded by a random vector $X$ with $E\|X\|^{2}<\infty$. Then, for any $\delta>(1 / 2)$, (11) holds.

Proof. By Lemma 3, Hölder's inequality, $E\|X\|^{2}<\infty$, the proof of Theorem 2, and Lemma 5 (i), we obtain

$$
\begin{aligned}
E\left(n^{-\delta} \max _{1 \leq i \leq n}\left\|S_{i}-E S_{i}\right\|\right)^{2} & \leq n^{-2 \delta} \sum_{i=1}^{n} E\left\|X_{i}\right\|^{2} \\
& \leq C n^{-2 \delta+1} E\|X\|^{2} \longrightarrow 0 \text { as } n \longrightarrow \infty .
\end{aligned}
$$

Hence, (11) holds.

We will extend two special kinds of uniform integrability which were introduced by Chandra and Goswami [14] to $H$-valued random vectors.

Definition 1 (see [15]). For $\alpha \in(0, \infty)$, a sequence $\left\{X_{n}, n \geq 1\right\}$ of random vectors in Hilbert space is said to be residually Cesàro alpha-integrable $(\mathrm{RCI}(\alpha))$ if 


$$
\begin{array}{r}
\sup _{n \geq 1} \frac{1}{n} \sum_{i=1}^{n} E\left\|X_{i}\right\|<\infty, \\
\lim _{n \longrightarrow \infty} \frac{1}{n} \sum_{i=1}^{n} E\left(\left\|X_{i}\right\|-i^{\alpha}\right) I\left(\left\|X_{i}\right\|>i^{\alpha}\right)=0 .
\end{array}
$$

Clearly, $\left\{X_{n}\right\}$ is RCI $(\alpha)$ for any $\alpha>0$ if $\left\{X_{n}, n \geq 1\right\}$ is identically distributed with $E\left\|X_{1}\right\|<\infty$ and $\left\{\left\|X_{n}\right\|^{2}, n \geq 1\right\}$ is RCI $(\alpha)$ for any $\alpha>0$ if $\left\{X_{n}, n \geq 1\right\}$ is stochastically dominated by a non-negative random vector $X$ with $E\|X\|^{2}<\infty$ (see [15]).

Theorem 4. Let a random vector $X_{n}=\left(X_{n}^{(1)}, X_{n}^{(2)}, \ldots, X_{n}^{(j)}\right)$ for any $j \geq 1$ be non-negative where every component $X_{n}^{(j)}$ of $X_{n}$ is non-negative random variable for each $j \geq 1$. Let $\left\{X_{n}, n \geq 1\right\}$ be a sequence of $H$-valued NSD non-negative random vectors. If $\left\{\left\|X_{n}\right\|^{2}, n \geq 1\right\}$ is RCI ( $\alpha$ ) for some $\alpha \in(0, \infty)$, then for any $\delta>(1 / 2)$,

$$
n^{-\delta} \max _{1 \leq i \leq n}\left\|S_{i}-E S_{i}\right\| \longrightarrow 0 \text { in } L_{2} \text {. }
$$

Proof. Let $j \geq 1 X_{n}=\left(X_{\eta}^{(1)}, X_{n}^{(2)}, \ldots, X_{n}^{(j)}\right)$ such that $X_{n}^{(j)} \geq$ 0 for all $j \geq 1$, where $X_{n}^{(\xi)}=\left\langle X_{n}, e_{j}\right\rangle$. For each $j \geq 1$, let

$$
\begin{aligned}
& Y_{n}^{(j)}=X_{n}^{(j)} I\left(0 \leq X_{n}^{(j)} \leq n^{\alpha}\right)+n^{\alpha} I\left(X_{n}^{(j)}>n^{\alpha}\right), \\
& Z_{n}^{(j)}=X_{n}^{(j)}-Y_{n}^{(j)}=\left(X_{n}^{(j)}-n^{\alpha}\right) I\left(X_{n}^{(j)}>n^{\alpha}\right) .
\end{aligned}
$$

Define, for each $n \geq 1 Z_{n}=X_{n}-Y_{n}, S_{n}^{(1)}=\sum_{i=1}^{n} Y_{i}$ and $S_{n}^{(2)}=\sum_{i=1}^{n} Z_{i}$. Then, $S_{n}=S_{n}^{(1)}+S_{n}^{(2)}=\sum_{i=1}^{n} Y_{i}+\sum_{i=1}^{n} Z_{i}$.

Note that $\left\{X_{n}^{(j)}\right\}$ is a sequence of NSD random variables and that

$$
\begin{aligned}
\left\|Y_{n}\right\| & =\min \left\{\left\|X_{n}\right\|, n^{\alpha}\right\}, \\
\left\|Z_{n}\right\| & =\left(\left\|X_{n}\right\|-n^{\alpha}\right) I\left(\left\|X_{n}\right\|>n^{\alpha}\right), \\
\left\|Z_{n}\right\|^{2} \leq & \left(\left\|X_{n}\right\|^{2}-n^{\alpha}\right) I\left(\left\|X_{n}\right\|^{2}>n^{\alpha}\right) .
\end{aligned}
$$

Obviously, for each $j \geq 1$ and $n \geq 1, Y_{n}^{(j)}$ and $Z_{n}^{(j)}$ are monotone transforms of the random variable $X_{n}^{(j)}$ by (16) and (17), respectively. Thus, $j \geq 1\left\{Y_{n}^{(j)}\right\}$ and $\left\{Z_{n}^{(j)}\right\}$ are NSD sequences of $H$-valued random variables by Lemma 1 . $\left\{Y_{n}^{(j)}-E Y_{n}^{(j)}\right\}$ and $\left\{Z_{n}^{(j)}-E Z_{n}^{(j)}\right\}$ are also NSD sequences of zero mean random variables. By Lemma 3 and the first condition of the RCI $(\alpha)$ property of (14) of the sequence $\left\{\left\|X_{n}\right\|^{2}, n \geq 1\right\}$, we obtain

$$
\begin{aligned}
n^{-2 \delta} E\left(\max _{1 \leq i \leq n}\left\|S_{i}^{(1)}-E S_{i}^{(1)}\right\|^{2}\right) & \ll n^{-2 \delta} \sum_{i=1}^{n} E\left\|Y_{i}-E Y_{i}\right\|^{2} \\
& \leq n^{-2 \delta} \sum_{i=1}^{n} E\left\|Y_{i}\right\|^{2} \leq n^{-2 \delta} \sum_{i=1}^{n} E\left\|X_{i}\right\|^{2} \\
& \leq n^{-2 \delta+1} \sup _{n \geq 1}\left(n^{-1} \sum_{i=1}^{n} E\left\|X_{i}\right\|^{2}\right) \\
& \longrightarrow 0 \text { as } n \longrightarrow \infty .
\end{aligned}
$$

By Lemma 3, the Hölder inequality and relation (19), and the second condition of the RCI $(\alpha)$ property (14) of the sequence $\left\{\left\|X_{n}\right\|^{2}, n \geq 1\right\}$, we obtain

$$
\begin{aligned}
& n^{-2 \delta} E\left(\max _{1 \leq i \leq n}\left\|S_{i}^{(2)}-E S_{i}^{(2)}\right\|^{2}\right) \ll n^{-2 \delta} \sum_{i=1}^{n} E\left\|Z_{i}-E Z_{i}\right\|^{2} \\
& \ll n^{-2 \delta} \sum_{i=1}^{n} E\left\|Z_{i}\right\|^{2} \\
& \leq n^{-2 \delta+1} \cdot \frac{1}{n} \sum_{i=1}^{n} E\left[\left(\left\|X_{i}\right\|^{2}-i^{\alpha}\right) I\right. \\
&\left.\cdot\left(\left\|X_{i}\right\|^{2}>i^{\alpha}\right)\right] \longrightarrow 0 \text { as } n \longrightarrow \infty .
\end{aligned}
$$

Thus, by (20) and (21), we have

$$
\begin{array}{r}
n^{-2 \delta} E\left(\max _{1 \leq i \leq n}\left\|S_{i}-E S_{i}\right\|^{2}\right) \\
\leq n^{-2 \delta} E\left(\max _{1 \leq i \leq n}\left\|S_{i}^{(1)}-E S_{i}^{(1)}\right\|^{2}\right) \\
+n^{-2 \delta} E\left(\max _{1 \leq i \leq n}\left\|S_{i}^{(2)}-E S_{i}^{(2)}\right\|^{2}\right) \longrightarrow 0 \text { as } n \longrightarrow \infty,
\end{array}
$$

which yields (15). The proof of theorem is completed.

Definition 2 (see [15]). For $\alpha \in(0, \infty)$, a sequence $\left\{X_{n}, n \geq 1\right\}$ of $H$-valued random vectors is said to be strongly residually Cesàro alpha-integrable $(\operatorname{SRCI}(\alpha))$ if

$$
\sup _{n \geq 1} \frac{1}{n} \sum_{i=1}^{n} E\left\|X_{i}\right\|<\infty
$$

$$
\sum_{n=1}^{\infty} \frac{1}{n} E\left(\left\|X_{n}\right\|-n^{\alpha}\right) I\left(\left\|X_{n}\right\|>n^{\alpha}\right)<\infty .
$$

Note that $\left\{\left\|X_{n}\right\|^{2}, n \geq 1\right\}$ is SRCI $(\alpha)$ for any $\alpha>0$, provided that $\left\{X_{n}, n \geq 1\right\}$ is stochastically dominated by a non-negative random vector $X$ with $E\|X\|^{2+\delta}<\infty$ for $\delta>0$.

Theorem 5. Define a $H$-valued non-negative random vector $X_{n}$ as in Theorem 4 Let $\left\{X_{n}, n \geq 1\right\}$ be a sequence of $H$-valued NSD non-negative random vectors. If $\left\{\left\|X_{n}\right\|^{2}, n \geq 1\right\}$ is SRCI $(\alpha)$ for some $\alpha \in(0, \infty)$, then for any $\delta>(1 / 2)$,

$$
n^{-\delta} \max _{1 \leq i \leq n}\left\|S_{i}-E S_{i}\right\| \longrightarrow 0 \text { completely. }
$$

Proof. For each $n \geq 1$, let $m=m_{n}$ be the integer such that $2^{m-1}<n \leq 2^{m}$. Then, we have that

$$
\begin{aligned}
n^{-\delta} \max _{1 \leq i \leq n}\left\|S_{i}-E S_{i}\right\| & \leq n^{-\delta} \max _{1 \leq i \leq 2^{m}}\left\|S_{i}-E S_{i}\right\| \\
& \leq\left(2^{m-1}\right)^{-\delta} \max _{1 \leq i \leq 2^{m}}\left\|S_{i}-E S_{i}\right\| \\
& \leq 2^{\delta} \cdot 2^{-m \delta} \max _{1 \leq i \leq 2^{m}}\left\|S_{i}-E S_{i}\right\| .
\end{aligned}
$$

It is sufficient to prove 


$$
2^{-m \delta} \max _{1 \leq i \leq 2^{m}}\left\|S_{i}-E S_{i}\right\| \longrightarrow 0 \text { completely. }
$$

Define $Y_{n}, Z_{n}, S_{n}^{(1)}$, and $S_{n}^{(2)}$ as in the proof of Theorem 4. To prove (26), we will first show that $2^{-m \delta} \max \left\|S_{i}^{(1)}-E S_{i}^{(1)}\right\| \longrightarrow 0$ completely. In other words, we willi ${ }^{1} 2^{m}$ rove

$$
2^{-m \delta} \max _{1 \leq i \leq 2^{m}}\left\|\sum_{k=1}^{i}\left(Y_{k}-E Y_{k}\right)\right\| \longrightarrow 0 \text { completely. }
$$

By Lemma 3 and the Hölder inequality, we have that

$$
\begin{aligned}
\sum_{m=0}^{\infty} E\left(2^{-m \delta} \max _{1 \leq i \leq 2^{m}}\left\|\sum_{k=1}^{i}\left(Y_{k}-E Y_{k}\right)\right\|\right)^{2} & \ll \sum_{m=0}^{\infty} 2^{-2 m \delta} \sum_{i=1}^{i=1} E\left\|Y_{i}\right\|^{2} \\
& \leq \sum_{m=0}^{\infty} 2^{-2 m \delta} \sum_{i=1}^{2^{m}} E\left\|X_{i}\right\|^{2} .
\end{aligned}
$$

In view of the first condition of the SRCI $(\alpha)$ property (23) of the sequence $\left\{\left\|X_{n}\right\|^{2}, n \geq 1\right\}$ and Lemma 4, we conclude that

$$
\begin{aligned}
\sum_{m=0}^{\infty} E\left(2^{-m \delta} \max _{1 \leq i \leq 2^{m}}\left\|\sum_{k=1}^{i}\left(Y_{k}-E Y_{k}\right)\right\|\right)^{2} & \ll \sum_{i=1}^{\infty} E\left\|X_{i}\right\|^{2} \sum_{\left\{m: 2^{m} \geq i\right\}} 2^{-2 m \delta} \\
& \leq \sum_{i=1}^{\infty} i^{-2 \delta} E\left\|X_{i}\right\|^{2} \leq\left(\sup _{n \geq 1} \frac{1}{n} \sum_{i=1}^{n} E\left\|X_{i}\right\|^{2}\right) \sum_{i=1}^{\infty} i^{-2 \delta}<\infty
\end{aligned}
$$

which yields (27). Next, we prove that

$$
2^{-m \delta} \max _{1 \leq i \leq 2^{m}}\left\|S_{i}^{(2)}-E S_{i}^{(2)}\right\| \longrightarrow 0 \text { completely. }
$$

Namely, we will prove that

$$
2^{-m \delta} \max _{1 \leq i \leq 2^{m}}\left\|\sum_{k=1}^{i}\left(Z_{k}-E Z_{k}\right)\right\| \longrightarrow 0 \text { completely. }
$$

By Lemma 3, the Hölder inequality, and (19) and the second condition of the SRCI $(\alpha)$ property (23) of the sequence $\left\{\left\|X_{n}\right\|^{2}, n \geq 1\right\}$, we have

$$
\begin{aligned}
\sum_{m=0}^{\infty} E\left(2^{-m \delta} \max _{1 \leq i \leq 2^{m}}\left\|\sum_{k=1}^{i}\left(Z_{k}-E Z_{k}\right)\right\|\right)^{2} & \ll \sum_{m=0}^{\infty} 2^{-2 m \delta} \sum_{i=1}^{2^{m}} E\left\|Z_{i}\right\|^{2} \\
& =\sum_{i=1}^{\infty} E\left\|Z_{i}\right\|^{2} \sum_{\left\{m: 2^{m} \geq i\right\}} 2^{-2 m \delta} \leq \sum_{i=1}^{\infty} i^{-2 \delta} E\left\|Z_{i}\right\|^{2} \\
& \leq \sum_{i=1}^{\infty} i^{-1} E\left\|Z_{i}\right\|^{2} \leq \sum_{i=1}^{\infty} i^{-1} E\left(\left\|X_{i}\right\|^{2}-i^{\alpha}\right) I\left(\left\|X_{i}\right\|^{2}>i^{\alpha}\right)<\infty,
\end{aligned}
$$

which yields (31). Thus, by (27) and (31), the desired result (26) follows. The proof of Theorem 5 is completed.

\section{Conclusions}

In this article, we obtain the maximal moment inequality for a sequence of $H$-valued NSD random vectors by extending the maximal moment inequality for a sequence of NSD random variables in Wang et al. [10] (see Lemma 3). Using this maximal moment inequality, we investigate the complete convergence results (see Theorem 1 ), $L_{2}$-convergence (see Theorems 2 and 3), and residual Cesàro alpha-integrability and strongly residual Cesàro alpha-integrability (see Theorems 4 and 5) for $H$-valued NSD random vectors.

\section{Data Availability}

No data were used to support this study.

\section{Conflicts of Interest}

The author declares that there are no conflicts of interest regarding the publication of this article.

\section{Acknowledgments}

This study was supported by Wonkwang University in 2020.

\section{References}

[1] K. Alam and K. M. L. Saxena, "Positive dependence in multivariate distributions," Communications in StatisticsTheory and Methods, vol. 10, no. 12, pp. 1183-1196, 1981, In press.

[2] J. H. B. Kemperman, "On the FKG-inequality for measures on a partially ordered space," Indagationes Mathematicae (Proceedings), vol. 80, no. 4, pp. 313-331, 1977. 
[3] T. Z. Hu, "Nagatively superadditive dependence of random variables with applications," Chinese Journal of Applied Probability and Statistics, vol. 16, pp. 133-144, 2000.

[4] T. C. Christofides and E. Vaggelatou, "A connection between supermodular ordering and positive/negative association," Journal of Multivariate Analysis, vol. 88, no. 1, pp. 138-151, 2004.

[5] M.-H. Ko, T.-S. Kim, and K.-H. Han, "A note on the almost sure convergence for dependent random variables in a Hilbert space," Journal of Theoretical Probability, vol. 22, no. 2, pp. 506-513, 2009.

[6] N. V. Huan, N. V. Quang, and N. T. Thuan, "Baum-Katz type theorems for coordinatewise negatively associated random vectors in Hilbert spaces," Acta Mathematica Hungarica, vol. 144, no. 1, pp. 132-149, 2014.

[7] M.-H. Ko, "Complete convergence for coordinatewise asymptotically negatively associated random vectors in Hilbert spaces," Communications in Statistics-Theory and Methods, vol. 47, no. 3, pp. 671-680, 2017.

[8] L. V. Dung, T. C. Son, and T. M. Cuong, "Weak laws of large numbers for weighted coordinatewise pairwise NQD random vectors in Hilbert spaces," Journal of the Korean Mathematical Society, vol. 56, no. 2, pp. 457-473, 2018.

[9] C. T. Son, M. T. Cuong, and L. V. Dung, "On the almost sure convergence for sums of negatively superadditive dependent random vectors in Hilbert spaces and its application," Communications in Statistics-Theory and Methods, vol. 49, no. 11, pp. 2770-2786, 2019.

[10] X. Wang, X. Deng, L. Zheng, and S. Hu, "Complete convergence for arrays of rowwise negatively superadditive-dependent random variables and its applications," Statistics, vol. 48, no. 4, pp. 834-850, 2014.

[11] D. M. Yuan and X. S. Wu, "Limiting behavior of the maximum of the partial sum for asymptotically negatively associated random variables under residual Cesàro alpha-integrability assumption," Journal of Statistical Planning and Inference, vol. 140, no. 9, pp. 2395-2402, 2010.

[12] A. Gut, "Complete convergence for arrays," Periodica Mathematica Hungarica, vol. 25, no. 1, pp. 51-75, 1992.

[13] P. L. Hsu and H. Robbins, "Complete convergence and the law of large numbers," Proceedings of the National Academy of Sciences, vol. 33, no. 2, pp. 25-31, 1947.

[14] T. K. Chandra and A. Goswami, "Cesàro $\alpha$-integrability and laws of large numbers-II," Journal of Theoretical Probability, vol. 19, no. 4, pp. 789-816, 2006.

[15] M. H. Ko, "Some strong laws of large numbers, $L_{2}$-convergence and complete convergence for $m$-AANA random vectors in Hilbert space," Stochastics, 2020. 